\title{
BMJ Open Practice patterns and outcomes associated with early sedation depth in mechanically ventilated patients: a systematic review protocol
}

\author{
Robert J Stephens, ${ }^{1}$ Matthew R Dettmer, ${ }^{2}$ Brian W Roberts, ${ }^{3}$ Susan A Fowler, ${ }^{4}$ \\ Brian M Fuller
}

To cite: Stephens RJ, Dettmer MR, Roberts BW, et al. Practice patterns and outcomes associated with early sedation depth in mechanically ventilated patients: a systematic review protocol. BMJ Open 2017;7:e016437. doi:10.1136/ bmjopen-2017-016437

- Prepublication history and additional material is published online only. To view please visit the journal online (http://dx.doi. org/10.1136/bmjopen-2017016437).

Received 15 February 2017

Revised 3 April 2017

Accepted 19 April 2017

\section{(1) CrossMark}

${ }^{1}$ Washington University School of Medicine, St Louis, Missouri, USA

${ }^{2}$ Emergency Services Institute, Respiratory Institute, Cleveland Clinic Foundation, Cleveland, Ohio, USA

${ }^{3}$ Department of Emergency Medicine, Cooper University Hospital, Camden, New Jersey, USA

${ }^{4}$ Bernard Becker Medical Library, Washington University, St Louis, Missouri, USA ${ }^{5}$ Departments of Emergency Medicine and Anesthesiology, Division of Critical Care Medicine, Washington University School of Medicine, St Louis, Missouri, USA

Correspondence to Dr Matthew R Dettmer; stephensr@wustl.edu

\section{ABSTRACT}

Introduction Mechanical ventilation is a commonly performed intervention in critically ill patients. Frequently, these patients experience deep sedation early in their clinical course. Emerging data suggest that the practice of early deep sedation may negatively impact patient outcomes. The purpose of this review is to assess the world's literature to describe and determine the impact of early deep sedation on the outcomes of mechanically ventilated patients.

Methods and analysis Randomised controlled trials and non-randomised studies will be eligible for inclusion in this systematic review. With the assistance of a medical librarian, we will comprehensively search MEDLINE, Embase, Scopus, Cochrane Central Register of Controlled Trials, Database of Abstracts of Reviews and Effects, and Cochrane Database of Systematic Reviews for peerreviewed literature. Grey literature from appropriate professional society conferences, held from 2010 to 2017, will be reviewed manually. Two authors will independently review all search results, and disagreements will be resolved through arbitration by a third author. If appropriate, meta-analysis will be used for quantitative analysis of the data. Heterogeneity between studies will be assessed using the $\mathrm{I}^{2}$ statistic.

Ethics and dissemination The proposed systematic review will not collect data that are associated with individual patients and does not require ethical approval. Results of this study will contribute to the understanding of early sedation, identify future research targets and guide early care in mechanically ventilated patients.

Trial registration number This systematic review has been registered in the international prospective register of systematic reviews (PROSPER0 \#CRD42017057264).

\section{INTRODUCTION}

Mechanical ventilation is a common intervention in critically ill patients. ${ }^{1}$ There is increasing recognition that the management of non-ventilator-related aspects of care is highly influential on outcome. The management of sedation plays a major role in the care of mechanically ventilated patients. ${ }^{2}$ While necessary to relieve pain and anxiety and

\section{Strengths and limitations of this study}

This is the first systematic review specifically studying the impact of early sedation depth on patient important outcomes.

- In preparation of this protocol we followed the Preferred Reporting Items for Systematic Reviews and Meta-Analysis Protocols guidelines, and our study is registered with the international prospective register of systematic reviews (PROSPERO).

- Our robust search strategy will decrease our risk of missing relevant studies.

- Our inclusion of non-randomised trials increases the risk of study bias.

improve tolerance of mechanical ventilation, sedatives have adverse effects on important patient-centred outcomes, such as lengths of stay, delirium and mortality. ${ }^{34}$ Present guidelines recommend that sedatives be titrated to achieve light, as opposed to deep, levels of sedation. ${ }^{2}$

Despite these recommendations, deep sedation in the intensive care unit (ICU) is common. Specifically, early deep sedation (ie, during the first 48 hours following initiation of mechanical ventilation) occurs in up to $76 \%$ of patients. ${ }^{56}$ An emerging body of research suggests that the level of sedation during this early time period is an independent predictor of patient outcomes. ${ }^{78}$ However, the bulk of prior sedation research has not been devoted to this initial early period, often not enrolling patients until after 48 hours of mechanical ventilation. ${ }^{6}$ While the available evidence supports maintenance of early light sedation, the strength of the association remains unclear.

There have been no systematic reviews on the impact of early sedation on clinical outcomes. An important next step for investigating early sedation practices is to analyse 
the world literature to ascertain the true impact of early sedation. In this systematic review, we seek to (1) describe the state of global literature focusing on early sedation; and (2) quantify the impact of early sedation depth on patient-centred outcomes. We hypothesise that deep sedation in the 48 hours period following initiation of mechanical ventilation will be associated with increased mortality and longer lengths of stay.

\section{METHODS AND ANALYSIS}

\section{Protocol and registration}

This systematic review protocol is prepared in accordance with the Preferred Reporting Items for Systematic Reviews and Meta-Analysis Protocols (PRISMA-P) statement (see online supplementary material 1). ${ }^{9} 10$ The final results will be reported according to the PRISMA statement and the Meta-analysis of Observational Studies in Epidemiology guidelines. ${ }^{11} 12$ Any deviation from the protocol will be reported with the final results, along with a rationale for protocol deviation. This study will be conducted starting in February 2017 with an intended completion date in May 2017. This systematic review has been registered in the international prospective register of systematic reviews (PROSPERO \#CRD42017057264).

\section{Search for and identification of studies}

An electronic search will include the following databases: MEDLINE, Embase, Scopus, Cochrane Central Register of Controlled Trials, Database of Abstracts of Reviews and Effects, and Cochrane Database of Systematic Reviews. The search terms include the concepts of mechanical ventilation, sedation depth, critical illness and outcome measures (including mortality, delirium, length of stay, tracheostomy, and time ventilated). These strategies were established using a combination of standardised terms and keywords. The fully reproducible search strategy is provided in online supplementary material 2 . The search was designed in cooperation with a medical librarian, who performed the electronic search.

The reference lists of the articles selected for inclusion will be manually screened to identify additional studies. To identify potential unpublished data, abstracts from the following meetings (from 2010 to 2017) will be manually searched: Society of Critical Care Medicine, European Society of Intensive Care Medicine, International Symposium on Intensive Care and Emergency Medicine, American Thoracic Society, Society for Academic Emergency Medicine, Pharmacotherapy, American Society of Anesthesiologists, European Society of Anaesthesiology, International Anesthesia Research Society, Trauma, Critical Care \& Acute Care Surgery, American Association for the Surgery of Trauma, and the Eastern Association for the Surgery of Trauma. An online search for details of clinical trials registration (ClinicalTrials.gov) will also be conducted to identify completed, but not yet published, clinical studies. The principal investigators of published

\begin{tabular}{|c|c|c|}
\hline & Inclusion criteria & Exclusion criteria \\
\hline Population & Age $\geq 18$ years & Age $<18$ years \\
\hline Intervention & $\begin{array}{l}\text { Invasive positive } \\
\text { pressure ventilation }\end{array}$ & Chronic ventilation \\
\hline $\begin{array}{l}\text { Reference } \\
\text { standard }\end{array}$ & $\begin{array}{l}\text { Objective measure of } \\
\text { sedation depth }\end{array}$ & None \\
\hline Outcomes & $\begin{array}{l}\text { Mortality } \\
\text { Hospital length of stay } \\
\text { Intensive care unit } \\
\text { length of stay } \\
\text { Time to extubation } \\
\text { Delirium } \\
\text { Incidence of } \\
\text { tracheostomy }\end{array}$ & None \\
\hline Study design & $\begin{array}{l}\text { Randomised controlled } \\
\text { trials } \\
\text { Prospective cohort } \\
\text { studies } \\
\text { Retrospective cohort } \\
\text { studies } \\
\text { Cross-sectional studies } \\
\text { Before-after trials }\end{array}$ & $\begin{array}{l}\text { Correspondences } \\
\text { Editorials } \\
\text { Non-human } \\
\text { studies }\end{array}$ \\
\hline
\end{tabular}

and unpublished studies will also be contacted as needed for clarification of potential data for inclusion.

\section{Eligibility criteria}

Studies will be eligible regardless of language and will include adult patients receiving invasive positive pressure ventilation. Randomised controlled trials (RCTs), as well as non-randomised studies (prospective and retrospective cohort analyses, cross-sectional studies, before-after trials), will be included. Non-randomised studies will be included for the following reasons: (1) a likelihood that the question of interest may not be investigated strictly with RCTs secondary to a lack of existing randomised trials; (2) to provide an explicit evaluation of strengths and weaknesses of the current literature; (3) to assess evidence of effects (benefit and harm); and (4) to provide evidence for the undertaking of randomised trials. Papers that are reviews, correspondences, editorials and non-human studies will be excluded. Eligibility criteria are listed in table 1.

The intervention will be the sedation provided during the first 48 hours of mechanical ventilation. The comparison will be sedation depth (light sedation vs deep sedation). Eligible studies must report some objective measure of sedation depth, such as the Richmond Agitation-Sedation Scale or the Glasgow Coma Scale. The clinical outcomes will be assessed according to sedation depth. These include mortality, delirium, ventilator-free days, hospital and ICU lengths of stay, and incidence of tracheostomy. The drugs used for early sedation will also be qualitatively reported, as will the study location (ie, ICU, emergency department). If there is a relative paucity of data describing early sedation, we will also qualitatively 
report the sedation provided at trial enrolment for RCTs. Similarly, we will report the depth of sedation at the time of trial enrolment for RCTs.

\section{Study selection and data abstraction}

Two independent reviewers will screen titles and abstracts of identified studies for eligibility. After this relevance screen, the two reviewers will compare their included studies to determine if disagreement exists. In cases of disagreement, the opinion of a third reviewer will be sought and a consensus will be reached. Full-text articles will then be obtained and these manuscripts will be reviewed for potential inclusion.

The same two reviewers will extract data using standardised forms. The following data on study characteristics will be collected and placed in a table: author, year of publication, study design, number of patients included, characteristics of the patient population, sedation data, study quality, risk of bias and outcomes. We will include pertinent study-specific comments in the table as needed.

\section{Assessment of study quality}

We will assess quality of randomised clinical trials using the Cochrane Collaboration's tool for assessing the risk of bias in clinical trials and report a summary assessment for the risk of bias for each studied outcome. ${ }^{13}$ For studies of observational design, quality will be assessed with the Newcastle Ottawa Scale, assigning a maximum of nine points. Five or fewer points will indicate a high risk of bias. ${ }^{14}$

\section{Assessment of publication bias}

A graphical display (funnel plot) of the size of the treatment effect against the precision of the trial will be used to evaluate for potential publication bias.

\section{Strategy for data synthesis}

We will provide a comprehensive narrative synthesis and qualitative analysis of the data, structured around outcomes related to sedation. After conducting the systematic review, if the data can be pooled, we will use a meta-analytic approach to quantitatively analyse the data. A random-effects model will be used to calculate pooled effect sizes and corresponding $95 \%$ CIs between deep and light sedation groups. ORs will be calculated for binary data, such as mortality comparisons. Continuous outcomes will be reported as mean difference, and overall effect estimates will be generated using a $\mathrm{Z}$ test and presented as mean differences. A p value of $<0.05$ will be considered statistically significant.

Heterogeneity between studies will be assessed using the $\mathrm{I}^{2}$ statistic, which will be reported as a point estimate with 95\% CIs. We will interpret this statistic using suggested thresholds for low $(25 \%-49 \%)$, moderate $(50 \%-74 \%)$ and high $(\geq 75 \%)$ values. ${ }^{15}$

We will perform sensitivity and subgroup analyses if the systematic review suggests that this is feasible and warranted to explore heterogeneity between studies.

\section{ETHICS AND DISSEMINATION}

As this is a systematic review of completed studies, no ethical approval will be required. Results from this systematic review will be submitted for publication in peer-reviewed journals and will be presented at national meetings.

This study will refine the understanding of the impact of early sedation practices and inform healthcare workers providing care to mechanically ventilated patients. We anticipate that this information will improve the postintubation care received by mechanically ventilated patients.

Over the past decade, there has been an increasing recognition that early advanced care has significant impact on patient outcome during critical illness. This concept has been shown to be true with regard to antibiotics in sepsis, lung protective ventilation in respiratory failure and reperfusion therapy in cerebrovascular accident. ${ }^{16-19}$ However, there has not been a similar focus on early sedation care for patients treated with mechanical ventilation.

This systematic review will provide a complete synopsis of the world's literature examining the impact of early deep sedation on patient outcomes, including mortality and lengths of stay. We will assess the cohort of studies for study quality, publication bias and heterogeneity, and determine if a meta-analysis is appropriate. We expect to find that early deep sedation is associated with worse mortality, longer lengths of stay and greater ventilation duration. Furthermore, we will identify knowledge gaps in the literature as future research targets.

In conclusion, this systemic review will aim to characterise and quantify the impact of early sedation on patient important outcomes. We hope this study yields additional evidence to guide clinical practice in mechanically ventilated patients, as well as targets for future investigation.

Contributors RJS: Study conception and design, drafting the manuscript, critical revision, guarantor of the review. MRD: Study design, drafting the manuscript, critical revision. BWR: Study design, drafting the manuscript, critical revision. SAF: Study design, devising the search strategy. BMF: Study conception and design, drafting the manuscript, critical revision.

Funding RJS received funding from Washington University Institute of Clinical and Translational Sciences Grant UL1 TR000448, subaward TL1 TR000449, from the National Center for Advancing Translational Sciences (NCATS) of the National Institutes of Health (NIH). BMF was funded by the KL2 Career Development Award, and this research was supported by Washington University Institute of Clinical and Translational Sciences (Grants UL1 TR000448 and KL2 TR000450) from the National Center for Advancing Translational Sciences (NCATS) of the National Institutes of Health (NIH). BMF was also funded by the Foundation for BarnesJewish Hospital Clinical and Translational Sciences Research Program (Grant \#8041-88). BWR was supported by a grant from the National Institutes of Health/ National Heart, Lung, and Blood Institute (K23HL126979).

\section{Competing interests None declared.}

Provenance and peer review Not commissioned; externally peer reviewed.

Open Access This is an Open Access article distributed in accordance with the Creative Commons Attribution Non Commercial (CC BY-NC 4.0) license, which permits others to distribute, remix, adapt, build upon this work non-commercially, and license their derivative works on different terms, provided the original work is properly cited and the use is non-commercial. See: http://creativecommons.org/ licenses/by-nc/4.0/ 
(c) Article author(s) (or their employer(s) unless otherwise stated in the text of the article) 2017. All rights reserved. No commercial use is permitted unless otherwise expressly granted.

\section{REFERENCES}

1. Wunsch $\mathrm{H}$, Linde-Zwirble WT, Angus DC, et al. The epidemiology of mechanical ventilation use in the United States. Crit Care Med 2010;38:1947-53.

2. Barr J, Fraser GL, Puntillo K, et al. Clinical practice guidelines for the management of pain, agitation, and delirium in adult patients in the intensive care unit. Crit Care Med 2013;41:263-306.

3. Pandharipande $P$, Cotton BA, Shintani A, et al. Prevalence and risk factors for development of delirium in surgical and trauma intensive care unit patients. J Trauma 2008;65:34-41.

4. Ouimet S, Kavanagh BP, Gottfried SB, et al. Incidence, risk factors and consequences of ICU delirium. Intensive Care Med 2007;33:66-73.

5. Shehabi Y, Bellomo R, Reade MC, et al. Early intensive care sedation predicts long-term mortality in ventilated critically ill patients. Am J Respir Crit Care Med 2012;186:724-31.

6. Shehabi Y, Chan L, Kadiman S, et al. Sedation depth and longterm mortality in mechanically ventilated critically ill adults: a prospective longitudinal multicentre cohort study. Intensive Care Med 2013;39:910-8.

7. Shehabi Y, Bellomo R, Reade MC, et al. Early intensive care sedation predicts long-term mortality in ventilated critically ill patients. Am J Respir Crit Care Med 2012;186:724-31.

8. Tanaka LM, Azevedo LC, Park M, et al. Early sedation and clinical outcomes of mechanically ventilated patients: a prospective multicenter cohort study. Crit Care 2014;18:R156.

9. Moher D, Shamseer L, Clarke M, et al. Preferred reporting items for systematic review and meta-analysis protocols (PRISMA-P) 2015 statement. Syst Rev 2015;4:1.
10. Shamseer L, Moher D, Clarke M, et al. Preferred reporting items for systematic review and meta-analysis protocols (PRISMA-P) 2015: elaboration and explanation. BMJ 2015;349:g7647.

11. Foy R. Preferred Reporting items for systematic reviews and MetaAnalyses: the PRISMA Statement. Annals of Internal Medicine 2010;151:264-9.

12. Stroup D, Berlin J, Morton S, et al. Meta-analysis of Observational studies in Epidemiology: a proposal for reporting. JAMA 2000;283:2010-2.

13. Cochrane Handbook for Systematic Reviews of Interventions. In: Higgins J, Green S, eds. Www.handbook.cochrane.org: the Cochrane Collaboration, 2011.

14. Wells G, Shea B, O'Connell D, et al. The Newcaste-Ottawa Scale (NOS) for assessing the quality of nonrandomised studies in metaanalyses 2008. http://www.ohri.ca/programs/clinical_epidemiology/ oxford.asp (accessed 02/02 2017).

15. Higgins JP, Thompson SG. Quantifying heterogeneity in a metaanalysis. Stat Med 2002;21:1539-58.

16. Tissue plasminogen activator for acute ischemic stroke. the National Institute of neurological disorders and Stroke rt-PA Stroke Study Group. N Engl J Med 1995;333:1581-7.

17. Fuller BM, Ferguson IT, Mohr NM, et al. A Quasi-Experimental, Before-After Trial examining the impact of an Emergency Department Mechanical Ventilator Protocol on Clinical outcomes and LungProtective ventilation in Acute Respiratory distress syndrome. Crit Care Med 2017;45:645-52.

18. Fuller BM, Ferguson IT, Mohr NM, et al. Lung-Protective ventilation initiated in the Emergency Department (LOV-ED): A QuasiExperimental, Before-After Trial. Ann Emerg Med 2017.

19. Kumar A, Roberts D, Wood KE, et al. Duration of hypotension before initiation of effective antimicrobial therapy is the critical determinant of survival in human septic shock. Crit Care Med 2006;34:1589-96. 